\title{
Is the Red Queen Sitting on the Throne?
}

\author{
Current Trends and Future Developments in \\ Human Health Research Regulation
}

\author{
Stuart G. Nicholls
}

\subsection{INTRODUCTION}

Human health research is a vast enterprise; worldwide, hundreds of billions of dollars are spent annually on health research involving millions of research participants. ${ }^{1}$ This research is guided by multiple regulations and guidance documents that commonly reflect several core principles: the protection of the rights and welfare of individual research participants; the promotion of justice in the practice and outcomes of research; and that human health research should be socially valuable (see van Delden and van der Graff, Chapter 4, in this volume). In addition, oftcited goals of health research regulation include the development of a culture of ethical concern among researchers and institutions, and the maintenance of public trust in the research enterprise. $^{2}$

However, these generally accepted principles belie an ongoing tension between the protection of individual participants through appropriate regulation, and the facilitation of health research. ${ }^{3}$ Authors have, for example, written regarding the amount of waste in research, including inefficient research regulation and management. ${ }^{4}$ Others have pointed to the variation in decisions $s^{5}$ and time taken - with associated costs ${ }^{6}$ - of obtaining ethics

${ }^{1}$ L. Shamseer et al., 'Improving the Reporting and Usability of Research Studies', (2013) Canadian Journal of Anaesthesia, 6o(4), 337-339; M. R. Macleod et al., 'Biomedical Research: Increasing Value, Reducing Waste', (2014) Lancet, 383 (9912), 101-104; I. Chalmers and P. Glasziou, 'Avoidable Waste in the Production and Reporting of Research Evidence', (2009) Lancet, 374(9683), 86-89; Food and Drug Administration, '2015-2016 Global Participation in Clinical Trials Report', (FDA, 2017); R. A. English et al., Transforming Clinical Research in the Unites States. Challenges and Opportunities 2010 Workshop Summary (Washington DC: The National Academies Press, 2010).

${ }^{2}$ H. F. Lynch et al., 'Of Parachutes and Participant Protection: Moving Beyond Quality to Advance Effective Research Ethics Oversight,' (2018) Journal of Empirical Research on Human Research Ethics, 14(3), 190-196.

3 E. Cave and C. Nichols, 'Reforming the Ethical Review System: Balancing the Rights and Interests of Research Participants with the Duty to Facilitate Good Research', (2007) Clinical Ethics, 2(2), 74-79; E. S. Dove, Regulatory Stewardship of Health Research: Navigating Participant Protection and Research Promotion (Cheltenham: Edward Elgar, 2O2O).

4 D. Moher et al., 'Increasing Value and Reducing Waste in Biomedical Research: Who's Listening?', (2016) Lancet, $3^{87}$ (10027), 1573-1586.

5 E. Angell et al., 'Consistency in Decision Making by Research Ethics Committees: A Controlled Comparison', (2006) Journal of Medical Ethics, 32(11), 662-664; E. L. Angell et al., "Is "Inconsistency" in Research Ethics Committee Decision-Making Really a Problem? An Empirical Investigation and Reflection', (2007) Clinical Ethics, 2(2), 92-99; G. Silberman and K. L. Kahn, 'Burdens on Research Imposed by Institutional Review Boards: The State of the Evidence and its Implications for Regulatory Reform,' (2011) Milbank Quarterly, 89(4), 599-627.

6 A. Chakladar et al., 'Paper Use in Research Ethics Applications and Study Contact', (2011) Clinical Medicine, 11(1), 44-47; A. G. Barnett et al., 'The High Costs of Getting Ethical and Site-Specific Approvals for Multi-Centre Research', 
approval. This criticism has led to increased focus on the efficiency and effectiveness of human research regulation. ${ }^{7}$

In this chapter, I highlight areas that have and, I suggest, will continue to stretch health research regulation, requiring the regulatory infrastructure to adapt and evolve in order to be both effective and efficient. In doing so, I point to changes in risk assessment considerations, underlying trends toward harmonisation and streamlining of research regulation, and alternative approaches to consent. However, I also highlight countertrends that may serve to undermine these changes. Thus, like the red queen in Lewis Carroll's Through the Looking-Glass, I propose that the health research regulatory system runs and runs as fast as it can, only to remain in the same place.

\subsection{ADAPTATION TO THE ENVIRONMENT: THE CHANGING RESEARCH LANDSCAPE}

The evolution of the research regulatory landscape is shaped by new technologies and research approaches. In this next section, I consider several areas that have pushed, and I suggest will continue to push, the boundaries of human research regulation, namely: increasingly diverse and multijurisdictional research; Big Data and artificial intelligence in health research; the learning healthcare system, and; emergency/disaster research.

While multisite studies are by no means novel, ${ }^{8}$ the scale of research has exploded, with a proliferation of data repositories, biobanks and other sources of data (see Shabani et al., Chapter 19, this volume). It is now common for research to routinely cross jurisdictional boundaries, with consequent variability in experiences with regulations that exist. ${ }^{9}$ This has prompted discussion of ways to facilitate and improve the regulation and oversight of multijurisdictional research, ${ }^{10}$ including the development of data sharing structures. ${ }^{11}$

However, the size of data is just one element. Research is now generating new types of data that stretch existing regulations. Social media, smartphones and wearable technology are being used as sources of data. Changes in hardware have been accompanied with rapid developments in analytic methods with a variety of Artificial Intelligence (AI) and other computationally heavy approaches (see Ho, Chapter 28, in this volume). These Big Data approaches have raised

(2016) Research Integrity and Peer Review, 1(1); M. D. Neuman et al., 'Time to Institutional Review Board Approval with Local versus Central Review in a Multicenter Pragmatic Trial', (2018) Clinical Trials, 15(1), 107-111; S. A. Page and J. Nyeboer, 'Improving the Process of Research Ethics Review', (2017) Research Integrity and Peer Review, 2(1).

7 R. Ashcroft et al., 'Reforming Research Ethics Committees', (2005) BMJ, 331(7517), 587-588; L. Abbott and C. Grady, 'A Systematic Review of the Empirical Literature Evaluating IRBs: What We Know and What We Still Need to Learn', (2011) Journal of Empirical Research on Human Research Ethics, 6(1), 3-19; P. Friesen et al., 'Of Straws, Camels, Research Regulation, and IRBs', (2019) Therapeutic Innovation \& Regulatory Science, 53(4), 526-534.

8 C. Grady, 'Institutional Review Boards: Purpose and Challenges', (2015) Chest, 148(5), 1148-1155.

9 Australian Clinical Trials Alliance (ACTA), 'Report on the 2014 National Summit of Investigator-Initiated Clinical Trials Networks', (ACTA, 2014); J. K. Alas et al., 'Regulatory Framework for Conducting Clinical Research in Canada', (2017) Canadian Journal of Neurological Sciences, 44(5), 469-474.

${ }^{10}$ E. S. Dove et al., 'Ethics Review for International Data-Intensive Research. Ad Hoc Approaches Mix and Match Existing Components', (2016) Science, 351(6280), 1399-1400; Grady, 'Institutional Review Boards'; S. G. Nicholls et al., 'Call for a Pan-Canadian Approach to Ethics Review in Canada', (2018) Canadian Medical Association Journal, 190(18), E553-E555.

${ }^{11}$ E. S. Dove et al., 'An Ethics Safe Harbour for International Genomics Research?', (2013) Genome Medicine, 5(11); E. S. Dove et al., 'Towards an Ethics Safe Harbor for Global Biomedical Research', (2014) Journal of Law and the Biosciences, 1(1), 3-51. 
questions about access to data and its use, ${ }^{12}$ as well the status of information generated by wearable devices ${ }^{13}$ and whether this constitutes health data that would be protected under privacy regulations.

Finally, the contexts of learning activities have raised fundamental questions about how different activities ought to be regulated and what oversight they should be subject to. Two examples of this are the learning healthcare system (LHS), in which research is incorporated into routine clinical practice and research within humanitarian crises, ${ }^{14}$ which has pushed regulatory and oversight processes due to the emergent and time-sensitive nature of the research (see Ganguli-Mitra and Hunt, Chapter 32 in this volume). While both of these activities blur research and practice, research conducted within disasters or humanitarian crises raise additional concerns regarding the inclusion of participants who are in a vulnerable position, the changing nature of risks in a time of crisis, and logistical issues in forming ethics review committees and conducting review in this context. ${ }^{15}$

\subsection{SUPPORTING SOCIALLY VALUABLE RESEARCH AND THE ROLE OF RISK}

In order to adapt to these changing environmental stressors, as well as the noted pressure from researchers to improve the efficiency of regulation and oversight, human health research regulations continue to evolve. Indeed, the vast increases in stored data and biological materials were an explicit driver for revisions to both the 2016 Council for International Organizations of Medical Sciences (CIOMS) International Ethical Guidelines for Healthrelated Research Involving Humans and the US Code of Federal Regulations, Title 45, Part 46 (45 CFR 46; herein referred to as the Common Rule), ${ }^{16}$ while research in humanitarian crises were also an addition to CIOMS 2016, prompted by experiences with Ebola and other humanitarian crises.

One aspect to these developments has been an evolution regarding how risk is assessed and responded to. In part, this is driven by greater attention to the perceived need to balance risk against the potential social value of research with historically under-researched populations, for example, pregnant women, children, or patients with co-morbidities, ${ }^{17}$ as discussed in the recent CIOMS revisions. The regulatory response to managing risks generated by research with traditionally under-researched or vulnerable populations - such as with disaster research - while supporting socially valuable research remains a live topic of debate. ${ }^{18}$

${ }^{12}$ C. Cath et al., 'Artificial Intelligence and the "Good Society": the USA, EU, and UK approach', (2018) Science and Engineering Ethics, 24(2), 505-528; B. Mittelstadt, 'Designing the Health-Related Internet of Things: Ethical Principles and Guidelines', (2017) Information, 8(3), 77.

13 P. P. O'Rourke et al., 'Harmonization and Streamlining of Research Oversight for Pragmatic Clinical Trials', (2015) Clinical Trials 12(5), 449-456.

${ }^{14}$ M. Jansse et al., 'Advances in Multi-Agency Disaster Management: Key Elements in Disaster Research', (2009) Information Systems Frontiers, 12(1), 1-7; M. Hunt et al. 'The Challenge of Timely, Responsive and Rigorous Ethics Review of Disaster Research: Views of Research Ethics Committee Members', (2016) PLoS One, 11(6); C. M. Tansey et al., 'Familiar Ethical Issues Amplified: How Members of Research Ethics Committees Describe Ethical Distinctions between Disaster and Non-Disaster Research', (2017) BMC Medical Ethics, 18(1).

15 S. Mezinska et al., 'Research in Disaster Settings: A Systematic Qualitative Review of Ethical Guidelines', (2016) BMC Medical Ethics, $17(1), 62$.

${ }^{16}$ E. A. Largent, 'Recently Proposed Changes to Legal and Ethical Guidelines Governing Human Subjects Research', (2016) Journal of Law and the Biosciences, 3(1), 206-216; J. J. van Delden and R. van der Graaf, 'Revised CIOMS International Ethical Guidelines for Health-Related Research Involving Humans', (2017) JAMA, 317(2), $135^{-136 . ~}$

${ }^{17}$ D. Schopper et al., 'Research Ethics Governance in Times of Ebola', (2017) Public Health Ethics, 10(1), 49-61.

${ }^{18}$ Hunt et al., 'The Challenge of Timely, Responsive and Rigorous Ethics Review'. 
Further, the concept of minimal risk is increasingly raised in the regulatory context; it is a precondition in the US regulations - both Common Rule and forthcoming US Food and Drug Administration (FDA) regulations - with respect to approvals for alteration of consent, and particularly a waiver of consent, as well as featuring in EU Clinical Trials Regulation. ${ }^{19}$ This will be particularly relevant for comparative effectiveness research within the LHS and where many interventions being assessed may be argued to be usual care and minimal risk.

Moreover, the LHS and disaster research blur the line between research and practice and several examples now exist where controversy has erupted over whether an ascribed activity would constitute research or not. ${ }^{20}$ Indeed, the LHS, disaster research, and secondary research use of data collected for routine clinical care problematise traditional frameworks for governance given the close approximation of activities to both research and practice. ${ }^{21}$ The blurring of the traditional distinction between research and care will continue to press regulations regarding the types of research that require regulatory approvals or ethics review, as well as the type and level of oversight they need.

\subsection{RESPONDING TO CRITICISM AND SUPPORTING RESEARCH: SIMPLIFYING AND HARMONISING PROCESSES}

A feature of many recent regulatory changes is the effort to reduce the burden from the research ethics review process in order to meet the efficiency demands of researchers. Changes in the USA reflect an ongoing trend of streamlining and harmonisation of ethics review structures and processes, which in some countries - such as the UK - have been ongoing over the last two decades. ${ }^{22}$ Other regulatory reform, such as the EU Directive 2001/20/EC on clinical trials, have sought to further harmonise approaches between jurisdictions. ${ }^{23}$

These changes attempt to both simplify approaches by exempting certain types of research from the need for ethics review - such as happened with changes to the US Common Rule and reduce the need for multiple review. Examples of such streamlining include changes in the Common Rule to implement a single Institutional Review Board (sIRB) as the board of record. Indeed, this is a common theme in many initiatives. ${ }^{24}$ In Canada, one such as example is the

19 European Union Clinical Trials Regulation 536/2014 [2014].

${ }^{2 \circ}$ N. E. Kass and P. J. Pronovost, 'Quality, Safety, and Institutional Review Boards: Navigating Ethics and Oversight in Applied Health Systems Research', (2011) American Journal of Medical Quality, 26(2), 157-159; D. A. Thompson et al., 'Variation in Local Institutional Review Board Evaluations of a Multicenter Patient Safety Study', (2012) Journal for Healthcare Quality, 34(4), 33-39; N. E. Kass et al., 'The Research-Treatment Distinction: A Problematic Approach for Determining Which Activities Should Have Ethical Oversight', (2013) Hastings Center Report, 43, S4-S15; D. Whicher and others, "The Views of Quality Improvement Professionals and Comparative Effectiveness Researchers on Ethics, IRBs, and Oversight', (2015) Journal of Empirical Research on Human Research Ethics, 10(2), 132-144.

${ }^{21}$ Kass et al., 'The Research-Treatment Distinction'; J. Piasecki and V. Dranseika, 'Research versus Practice: The Dilemmas of Research Ethics in the Era of Learning Health-Care Systems', (2019) Bioethics, 33(5), 617-624; J. Piasecki and V. Dranseika, 'Learning to Regulate Learning Healthcare Systems', (2019) Cambridge Quarterly of Healthcare Ethics, 28(2), 369-377; Schopper et al., 'Research Ethics Governance'.

${ }_{22}$ A. Hedgecoe et al., 'Research Ethics Committees in Europe: Implementing the Directive, Respecting Diversity', (2006) Journal of Medical Ethics, 32(8), 483-486; R. Al-Shahi Salman et al., 'Increasing Value and Reducing Waste in Biomedical Research Regulation and Management', (2014) Lancet, 383(9912), 176-185; E. S. Dove, 'Requiring a Single IRB for Cooperative Research in the Revised Common Rule: What Lessons Can Be Learned from the UK and Elsewhere?', (2019) Journal of Law, Medicine E Ethics, 47(2), 264-282.

${ }^{23}$ Hedgecoe et al., 'Research Ethics Committees in Europe'.

24 Nicholls et al., 'Call for a Pan-Canadian Approach to Ethics Review'. 
Ontario Cancer Research Ethics Board (OCREB), which serves as the single board of record for multicentre oncology trials for twenty-six of twenty-seven cancer research sites in the province. ${ }^{25}$

A second trend in recent regulatory changes has been to simplify consent requirements. Indeed, it is a longstanding point of discussion that written consent forms do not necessarily facilitate informed decision-making on the part of potential research participants ${ }^{26}$ due to their length and complexity of language. ${ }^{27}$ Recent changes to the US Common Rule have served to try and improve these consent processes ${ }^{28}$ and the FDA has sought to open up the possibility of waivers of consent to align with the Common Rule. ${ }^{29}$ Moreover, alternate forms of consent were also part of the recent CIOMS revisions, which provide guidance regarding consent approaches and alternatives to prospective individual written consent. ${ }^{30}$ Indeed, some authors have recently argued that in the context of standard-of-care comparative effectiveness trials - such as those envisioned within a LHS - consent could (or should) be waived. ${ }^{31}$

This trend of alternate consent models will, I propose, continue due to developments in disaster and emergency research and the LHS, where the need for timely and expedient consent approaches - or even waivers of consent - will continue the push for the simplification of, or alternative approaches to, consent processes. Meanwhile, Big Data applications and the secondary use of existing data will further press regulations regarding the types of research that require explicit consent from participants and how this is managed.

\subsection{COUNTERVAILING TRENDS}

Alongside these trends in the regulation of health research, there are emerging examples of regulatory or oversight processes which may run counter to those listed above and may serve to nullify the potential impact of streamlining initiatives or attempts to create a more efficient regulatory environment.

One such example has been the implementation of 'permission to contact' or 'consent to contact' policies. While such policies vary, they represent a broad consent approach to being contacted about future research and are generally asked of all patients upon arrival or intake at hospital, with the goal of expediting recruitment and easing the burden on clinical investigators by allowing researchers to contact potential patient participants. Despite the

${ }^{25}$ R. Saginur et al., 'Ontario Cancer Research Ethics Board: Lessons Learned from Developing a Multicenter Regional Institutional Review Board', (2008) Journal of Clinical Oncology, 26(9), 1479-1482; R. Saginur et al., 'Ethics Review of Multi-Centre Trials: Where Do We Stand?', (2009) Health Law Review, 17(2-3), 59-65.

${ }^{26}$ J. C. Brehaut et al., 'Informed Consent Documentation Necessary but Not Sufficient', (2009) Contemporary Clinical Trials, 30(5), 388-389; J. C. Brehaut et al., 'Informed Consent Documents Do Not Encourage Good-Quality Decision Making', (2012) Journal of Clinical Epidemiology, 65(7), 708-724; J. C. Brehaut et al., 'Using Decision Aids May Improve Informed Consent for Research', (2010) Contemporary Clinical Trials, 31(3), 218-220.

27 C. Grady, 'Enduring and Emerging Challenges of Informed Consent', (2015) New England Journal of Medicine, 372(9), 855-862; C. Grady, 'The Changing Face of Informed Consent', (2017) New England Journal of Medicine, $376(9), 856-859$.

${ }_{28}$ J. Menikoff et al., 'The Common Rule, Updated,' (2017) The New England Journal of Medicine, 376(7), 613-615.

${ }^{29}$ US Department of Health and Human Services, Food and Drug Administration, Office of Good Clinical Practice (OGCP), Center for Drug Evaluation and Research (CDER), Center of Biologics Evaluation and Research (CBER) and Center for Devices and Radiological Health (CDRH), 'IRB Waiver or Alteration of Informed Consent for Clinical Investigations Involving No More Than Minimal Risk to Human Subjects. Guidance for Sponsors, Investigators, and Institutional Review Boards', (2017).

$3^{\circ}$ Largent, 'Recently Proposed Changes'.

${ }^{31}$ R. Dal-Ré et al., 'Low Risk Pragmatic Trials Do Not Always Require Participants' Informed Consent', (2019) BMJ, 364 , $175^{6}-1833$. 
intuitive appeal, this may, in fact, increase administrative burden and create uncertainty and inefficiencies in research. For example, studies have suggested that patients may customise what aspects of their data they could be approached about or could limit elements, meaning further review of records would be needed..$^{32}$ Others have suggested that while uptake to specific research may increase among those giving permission to contact (compared to those recruited through traditional physician-mediated contacts), there may be significant differences in the age and gender of those who agreed compared to those who declined the permission to contact form. ${ }^{33}$

A further consideration is whether a permission to contact agreement constitutes a valid consent. Studies indicate that there may be confusion among patients as to whether they were immediately signing up to research, ${ }^{34}$ and this may be especially true when patients are recruited upon arrival to the hospital prior to diagnosis. ${ }^{35}$ Consequently, permission to contact approaches not only have the potential to increase administrative burden, thus potentially negating efficiencies created elsewhere, but also raise important questions as to the status of such permission and how it should be considered within the regulatory process.

Other inefficiencies may be introduced through additional regulatory committees. ${ }^{36}$ Friesen and colleagues, for example, note a multitude of committees that now commonly exist in the USA in addition to Institutional Review Board (IRB) review, with inconsistency in the policies and processes of many of these additional bodies. ${ }^{37} \mathrm{~A}$ recent study indicated that the requirement to complete multiple local governance reviews had the effect that overall times to the startup of research were no less than if multiple local IRB review - as opposed to sIRB review - had been used. ${ }^{38}$ As such, the introduction of additional governance and oversight structures appear to negate potential benefits generated through streamlining initiatives for research ethics review procedures.

\subsection{CONCLUSiON: A PARTing CALl FOR THE EVALUATION OF REGULATORY CHANGES}

To conclude, we appear to be moving toward an era in which research requires regulation to not only protect participants, but also to be responsive, adaptive and supportive of research; responsive in its ability to facilitate research that can address emerging and emergent topics; adaptive in so far as it is malleable and flexible enough to cope with the ever-changing technologies and data needs of health research; and supportive insofar as the regulatory responses facilitate socially valuable research with no more bureaucracy than is necessary. I have proposed several areas

${ }^{22}$ C. Papoulias et al., 'Staff and Service Users' Views on a "Consent for Contact” Research Register Within Psychosis Services: A Qualitative Study', (2014) BMC Psychiatry, 14(1), 377.

33 I. Druce et al., 'Implementation of a Consent for Chart Review and Contact and Its Impact in One Clinical Centre', (2015) Journal of Medical Ethics, 41(5), 425-428.

34 D. Robotham et al., 'Consenting for Contact? Linking Electronic Health Records to a Research Register Within Psychosis Services, a Mixed Method Study’, (2015) BMC Health Services Research, 15(1), 199.

35 A. S. Iltis, 'Timing Invitations to Participate in Clinical Research: Preliminary versus Informed Consent', (2005) Journal of Medicine and Philosophy, 30(1), 89-106.

${ }^{6}$ O'Rourke et al., 'Harmonization and Streamlining'; Friesen et al., 'Of Straws, Camels, Research Regulation, and IRBs.'

37 Friesen et al., 'Of Straws, Camels, Research Regulation, and IRBs.'

$3^{8}$ M. P. Diamond et al., 'The Efficiency of Single Institutional Review Board Review in National Institute of Child Health and Human Development Cooperative Reproductive Medicine Network-Initiated Clinical Trials', (2018) Clinical Trials, 16(1), 3-10. 
where research regulation will continue to evolve in the near future - streamlined review processes, a focus on the management of risk, and regulatory changes to facilitate and support alternatives to traditional written consent forms.

Yet we also need to be mindful of trends that may run counter to these and which may impede progress. The noted proliferation of additional committees seems to serve only to introduce more variation and regulatory hurdles. Viewed alongside attempts to streamline ethics review, this may be seen as giving with one hand while taking away with the other. Experience in the UK with the rise of research governance following the streamlining of ethics review should serve as a historic warning. ${ }^{39}$ Similarly, the introduction of permission to contact mechanisms may, in theory, lead to streamlined research by allowing researchers to directly contact patients - as opposed to mediated contact via physicians - who have opted into research, yet questions remain as to the status of such a consent as well as the practicalities of conducting a system which may well create the need for additional checks that could serve to increase administrative burden rather than decrease it. Based on the above, we appear doomed to repeat the failures of the past and despite running as fast as we can to make changes, we may ultimately stay in the same place.

This leads to my final parting call: the need for good evaluative research of changes to health research regulations and oversight systems. Despite all the discussion regarding changes to the regulatory landscape, this is largely an evidence-free space. Indeed, while the Notice of Proposed Rule Making issued in advance of changes to the Common Rule ran to $13^{1}$ pages, there was a distinct lack of evidence regarding how the proposed changes addressed purported deficiencies in the oversight of research. ${ }^{40}$ Moreover, there is a paucity of evidence regarding whether changes to sRB approaches have had the sought-after effect on review processes. Klitzman, for example, notes that the introduction of policies regarding the use of sIRBs has been done in the absence of the systematic collection of data', ${ }^{41}$ while Rahimzadeh and colleagues note that, as yet, one cannot point to rigorous evidence that the sIRB model offers great advantages over current practice. ${ }^{42}$

Without empirical evaluation, we will not know whether changes in regulation or oversight are having the desired effect, or whether they increase inefficiencies and convey no benefits to research participants. This potential is borne out by the handful of studies that do exist and which show that, despite a good theoretical basis for an intervention, some may not bring about the desired improvement. ${ }^{43}$ Thus, there is a need to develop strong theory-based

39 R. Al-Shahi, 'Research Ethics Committees in the UK - The Pressure Is Now on Research and Development Department', (2005) Journal of the Royal Society of Medicine, 98(10), 444-447; R.A-S. Salman et al., 'Research Governance Impediments to Clinical Trials: A Retrospective Survey', (2007) Journal of the Royal Society of Medicine, 100(2), 101-104; A. G. H. Thompson and E. F. France, 'One Stop or Full Stop? The Continuing Challenges for Researchers Despite the New Streamlined NHS Research Governance Process', (2010) BMC Health Services Research, $10(1), 124$.

$4^{\circ}$ D. H. Strauss et al., 'Reform of Clinical Research Regulations', (2016) New England Journal of Medicine, 374(17), 1693-1694; S. G. Nicholls, 'Revisions to the Common Rule: A Proposal in Search of Evidence', (2017) Research Ethics, $13(2), 92-96$.

${ }^{41}$ R. Klitzman et al., 'Single IRBs in Multisite Trials. Questions Posed by the New NIH Policy', (2017) JAMA, 317(20), 2061-2062.

$4^{2}$ V. Rahimzadeh et al., 'The sIRB System: A Single Beacon of Progress in the Revised Common Rule?', (2017) American Journal of Bioethics, $17(7), 43-46$.

43 M. Dixon-Woods et al., 'Can an Ethics Officer Role Reduce Delays in Research Ethics Approval? A mixed-Method Evaluation of an Improvement Project', (2016) BMJ Open, 6(8), eo11973; S. Sonne et al., 'Regulatory Support Improves Subsequent IRB Approval Rates in Studies Initially Deemed Not Ready for Review: A CTSA Institution's Experience', (2018) Journal of Empirical Research on Human Research Ethics, 13(2), 139-144. 
approaches, research designs that bring rigour to the evaluation of regulatory change and a culture change that views evidence-based approaches not with scepticism but with openness. ${ }^{44}$ Without the collection of data and open sharing of results, we cannot develop the necessary learning regulatory environment as envisaged and laid out at the outset to this collection.

44 S. G. Nicholls, 'Commentary on "Regulatory Support Improves Subsequent IRB/REC Approval Rates in Studies Initially Deemed Not Ready for Review: A CTSA Institution’s Experience”, (2018) Journal of Empirical Research on Human Research Ethics, 13(2), 145-147 